\title{
Chemo fog becomes clearer
}

It has been known for a long time that chemotherapy may have neurotoxic effects on cognitive functioning, an effect commonly known as 'chemo brain' or 'chemo fog'. However, despite the important repercussions of these observations, little research has been carried out about whether-and to what extent-chemotherapy affects long-term cognitive functioning. Several recent large controlled studies have added evidence supporting the association of chemotherapy treatment and cognitive deficits. Heather Jim and colleagues have now scrutinized this information in a meta-analysis of data obtained from 17 studies that included a total of 807 survivors of breast cancer who had been treated with standard-dose chemotherapy at least 6 months earlier, and 291 controls. They assessed the effect of chemotherapy in eight cognitive domains: attention, executive functioning, information processing, motor speed, verbal ability, verbal memory, visual memory, and visuo-spatial ability. The researchers found that patients who had been previously treated with chemotherapy performed significantly worse on tests of domains such as verbal and visuo-spatial ability, than patients who had not been treated with chemotherapy or did not have cancer, respectively. Interestingly, age and education did not seem to have an effect in chemotherapyinduced cognitive deficit. This metaanalysis clears any doubts about the existence of chemo fog, and calls for future studies to address this important issue.

\section{Teresa Villanueva}

Original article Jim, H. S. L. et al. Meta-analysis of cognitive functioning in breast cancer survivors previously treated with standard-dose chemotherapy. J. Clin. Oncol. doi:10.1200/JC0.2011.39.5640 